\title{
Characterization of a low consumption wireless sensor node for the intensive transmission of physiological signals
}

\author{
Ricardo Yauri ${ }^{1}$, Santiago Rubiños ${ }^{2}$, Juan Grados ${ }^{3}$, Mario Chauca ${ }^{4}$ \\ ${ }^{1}$ National Institute of Telecommunications Research and Training- National University of Engineering, Peru \\ ${ }^{2,3,4}$ Faculty of Electrical and Electronic Engineering, National University of Callao, Peru
}

\section{Article Info \\ Keywords \\ BAN \\ GPRS \\ Sensors \\ Wi-Fi \\ WSN \\ ZigBee}

Article history:

Received Sep 25, 2018

Revised Dec 27, 2018

Accepted Jan 15, 2019

\begin{abstract}
This paper describes the development and implementation of low power consumption wireless sensor nodes for the periodic monitoring of physiological signals with intensive data transmission, using $\mathrm{Wi}-\mathrm{Fi}$ and ZigBee wireless communication modules, obtaining operation characteristics from the energy point of view that allow to increase the life time of the sensor node. The sensor nodes are designed and built using low energy consumption electronic devices to evaluate their energy performance using current data, transmission time, data transmission period and the relationship with the sensor node's lifetime when transmitting electrocardiographic (ECG), temperature and pulse type physiological signals. In this way, results are obtained that can allow the data transmission period, current consumption and size of data sent to be related to the operating time, defining the operating conditions and wireless technologies that allow the optimization of energy consumption when data is sent to Internet monitoring applications.
\end{abstract}

Copyright (c) 2019 Institute of Advanced Engineering and Science. All rights reserved.

\section{Corresponding Author:}

Mario Chauca,

Faculty of Electrical and Electronic Engineering,

National University of Callao,

306 Juan Pablo II Avenue, Callao, Perú.

Email: mchauca@ulima.edu.pe

\section{INTRODUCTION}

The sensor nodes currently have multiple applications in various fields, aimed at providing solutions for industrial automation, military applications, medicine in rural environments, home automation or indoor location systems, where they are responsible for acquiring, collecting and centralizing information automatically and in places of difficult access. They are also small in size, with wireless communication, low in cost and low in energy consumption.

The energy efficiency in the wireless communication stage and its effect on the useful life of a sensor node is a subject of many research activities being ZigBee the most common technology in the implementation of sensor nodes, due to its communication range characteristics and low power consumption. On the other hand, the recent appearance of low power Wi-Fi modules in the market, allows to consider it as an option for the transmission of a large amount of data and has the facility to be integrated into existing communication networks.

The work developed in this article proposes an alternative solution to remote monitoring of patients through the implementation of wireless sensor nodes for the acquisition of electrocardiographic signals, temperature and pulse, and sending them to a Web application on the Internet. The design and implementation of sensor nodes is carried out considering the development of communication stages with ZigBee transmission technologies, WiFi and a GPRS Gateway (in external communication scenarios). 


\section{PROBLEM STATEMENT}

Wireless sensor nodes, used in periodic data intensive monitoring applications, require a particular design and implementation according to the types of signals to be transmitted, transmission period and power restrictions. The stage with the highest current consumption is during data transmission, which directly affects the lifetime of the sensor node. Therefore, the central problem of this work is the need to evaluate and test the energy performance of sensor nodes using ZigBee and Wi-Fi transmission technologies, which are commercially available in the local market, and to define their characteristics of use in low consumption conditions to increase their lifetime.

\section{THEORETICAL FOUNDATION THEORETICAL CONCEPTS}

\subsection{Wireless Body Area Networksl}

Wireless networks can be classified by their coverage, where we can find those that are used in extended area, metropolitan, local and personal. In the case of personal area networks, there are wireless sensor networks (WSN), the most commonly used technologies being: ZigBee, WiFi, Bluetooth and GPRS [1]. As part of the WSN we have the body area networks (BAN) which are used for monitoring medical signals and are composed of small nodes with physiological sensors that collect information from the human body and then transmit it to a monitoring or storage system. A schematic of the BAN networks is shown in Figure 1.

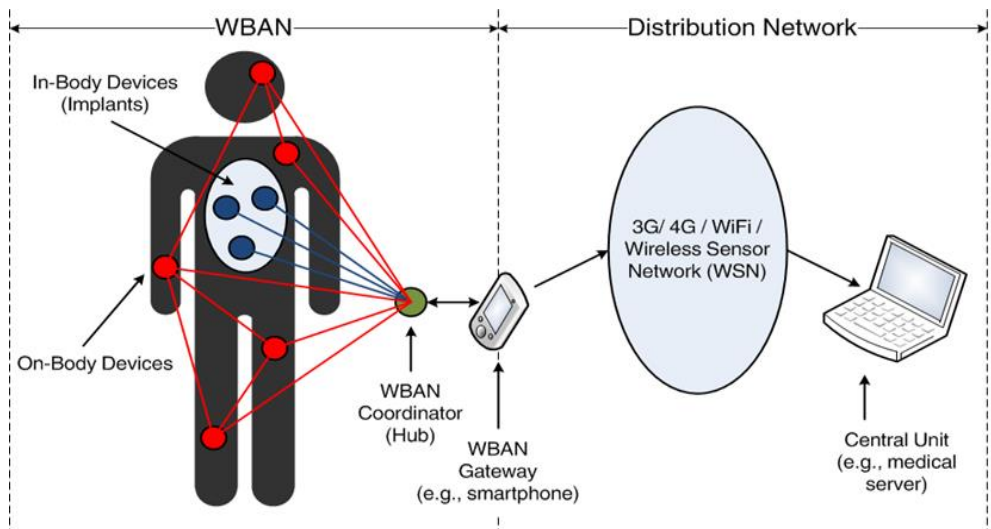

Figure 1. Body area sensor network [2]

\subsection{Wi-Fi}

For the selection of wireless communication devices, low power consumption and integration with microcontrollers in embedded systems is considered. The IEEE 802.11 standard defines the use of the two lower levels of the OSI architecture or model (physical layer and data link layer), specifying the operating rules of a wireless local area network (WLAN). When data transmission is intensive using Wi-Fi there is a high power consumption, but recently modules have been developed for use in applications with power limitations such as ESP8266, from the company Espressif Systems or ZigBee Technology. ESP8266 module offers a complete and economical solution, allowing to delegate all the functions related to the implementation of TCP/IP communication protocols to the processor [4]. These modules are shown in Figure 2.
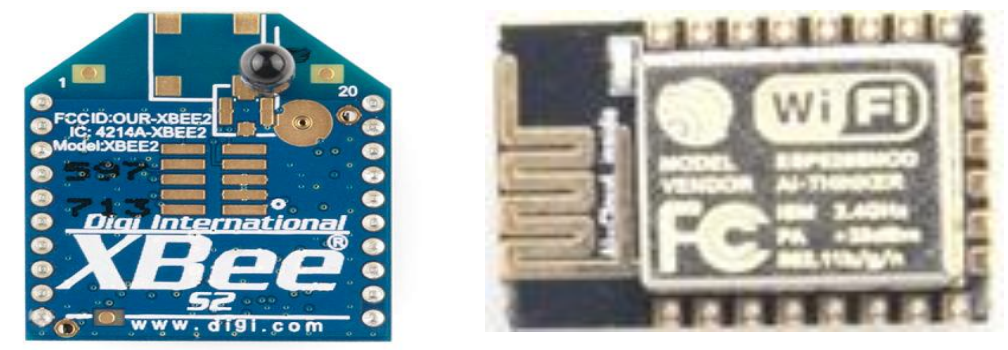

Figure 2. Xbee Module (left) [3] and WI-FI ESP12E (right) [4] 


\subsection{Physiological Signals}

In order to acquire and transmit physiological signals, the sampling rate, hardware limitations, power and processing speed must be considered. In this work, ECG, temperature and cardiac pulse signals will be used for the implementation of the sensor nodes. The electrocardiographic signal is represented as a record of cardiac activity [5] and its amplitude can reach up to $3 \mathrm{mV}$, but for implementations of a cardiac monitor it is possible to use frequencies lower than $30 \mathrm{~Hz}$ and this also applies to pulse signals, while in the case of temperature the frequency can be $1 \mathrm{~Hz}$.

\section{PROBLEM STATEMENT}

\subsection{Sensor Node}

The sensor node is designed with commercially available low-power, battery-powered components, considering the use of sleep mode and communication stage control. The blocks that make up the node are shown in Figure 3. The sensor block is responsible for acquiring one of the three signals defined in Table 1. In the case of the ECG signal, an AD8232 integrated circuit is used and designed to extract, amplify and filter out very low frequency noise and then acquire it with an ADC module. The electronic schematic of the AD8232 circuit is shown in Figure 4.

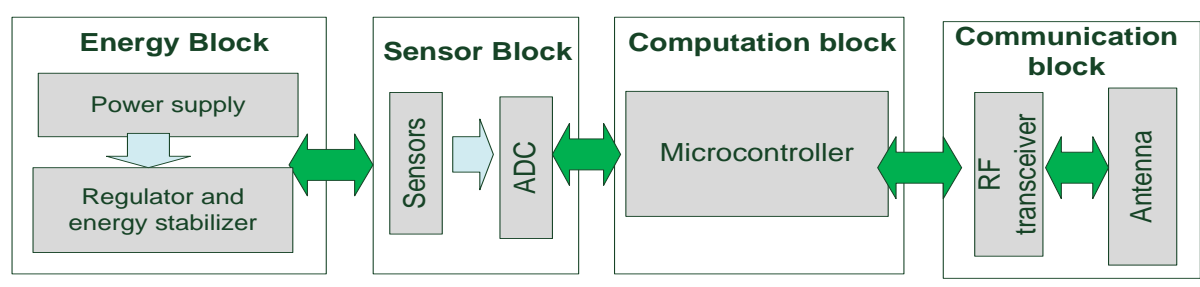

Figure 3. Blocks of a sensor node

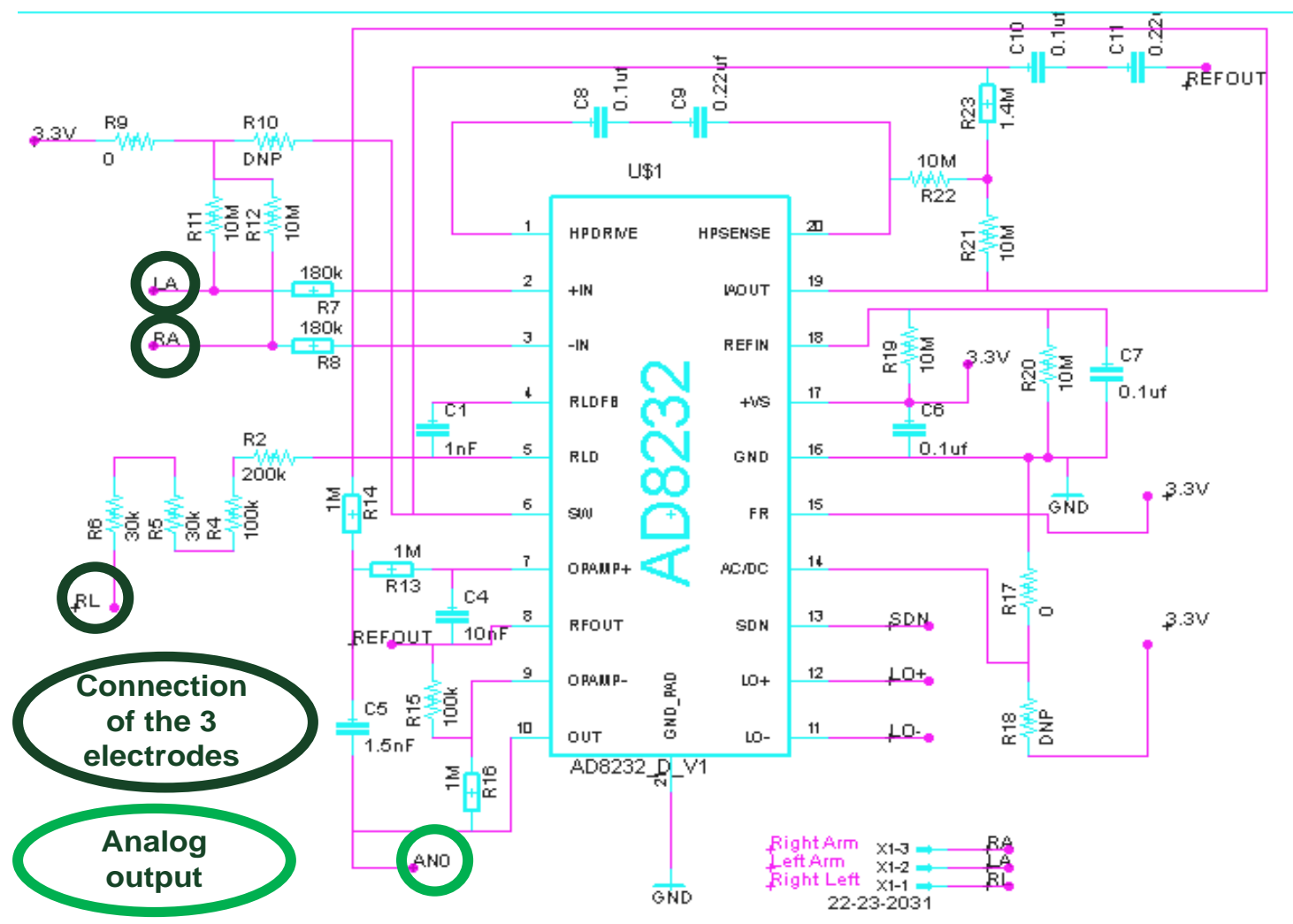

Figure 4. Electronic schematic of circuit AD8232 
The DS18B20 [7] digital sensor is used to acquire the temperature signal, using the One-Wire communication protocol. Finally, the analogue pulse sensor distributed by "World Famous Electronics llc" is used, which can be placed on the finger or ear. For the processing block, the microcontroller integrated in the ESP12E module is used, which controls the acquisition, processing and transmission of the signals.

The communication block uses the Wi-Fi modem integrated in ESP12E, while in Figure 5 the electronic schematic of the ESP8266 module is shown. The scheme of the three sensor nodes implemented is shown in Figure 6. The characteristics of the two transmission modules can be seen in Table 2.

Table 1. Frequency Range of Physiological Signals [6]

\begin{tabular}{cc}
\hline Parameter & Frequency range \\
\hline ECG & $0.5 \mathrm{~Hz}-100 \mathrm{~Hz}$ \\
Body temperature & $1 \mathrm{HZ}$ \\
Breathing & $10 \mathrm{~Hz}$ \\
Electromyograph & $10 \mathrm{~Hz}-5 \mathrm{KHz}$ \\
\hline
\end{tabular}
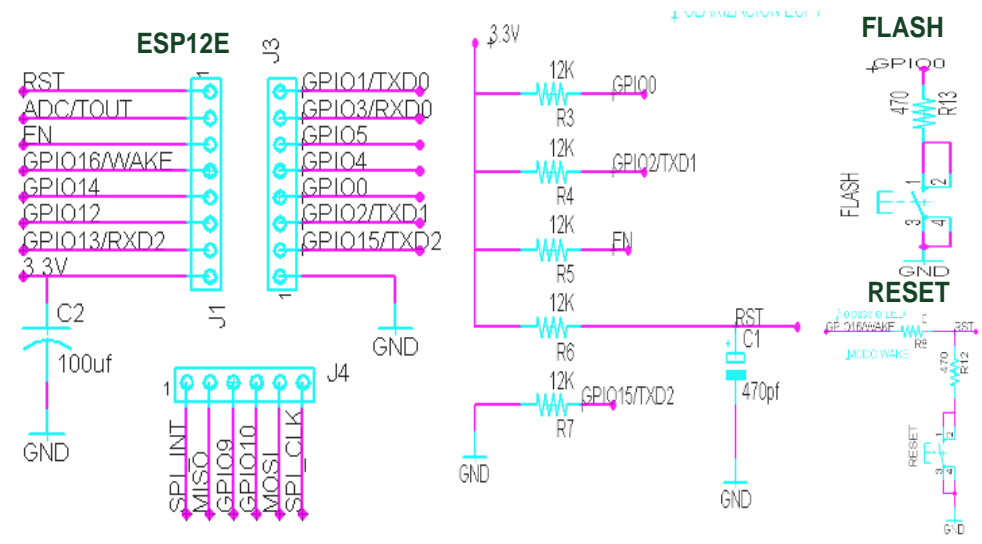

Figure 5. Schematic polarization of ESP12E

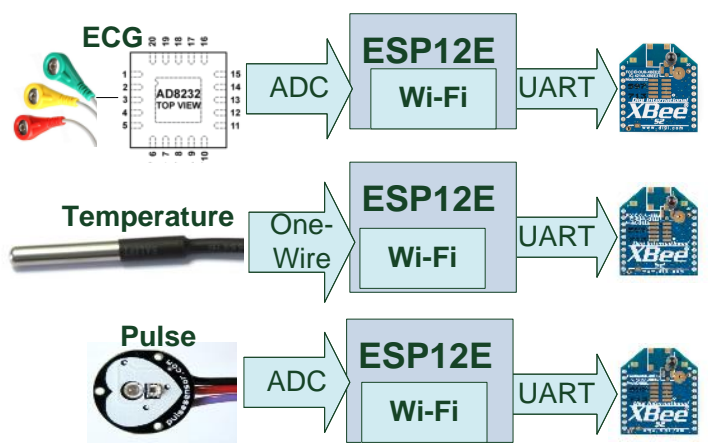

Figure 6. Sensor connection diagram and with ESP12E module on all three sensor nodes

Table 2. Characteristics of Wireless Modules

\begin{tabular}{ccc}
\hline & Xbee Series 2 & ESP8266 \\
Voltage supplied & 3.3 & 3.3 \\
\hline Transmission current $(\mathrm{mA})$ & $35-45$ & $120-170$ \\
Sleep Current $(\mathrm{mA})$ & $<0.001$ & $<0.01$ \\
Distance $(\mathrm{mt})$ & $40-120$ & $30-40$ \\
Transmission power $(\mathrm{dBm})$ & $1.25-2$ & $20(802.11 \mathrm{~b})$ \\
Transmission rate $(\mathrm{Mbps})$ & 0.25 & $1 / 11 / 54$ \\
Operating frequency $(\mathrm{GHz})$ & $2.4 \mathrm{GHz}$ & $2.4-2.5$ \\
Clock CPU $(\mathrm{MHz})$ & 50.33 & 80 \\
\hline
\end{tabular}


To coordinate the actions of the modules and components of the sensor node, a status machine is used that starts with the acquisition of signals to continue with the processing and transmission of the data. The sensor node always enters low power mode and disables other hardware modules after transmitting and storing the information in its internal memory. Finite state machine of the sensor node firmware shown in Figure 7. With the blocks defined, the hardware of the sensor node shown in Figure 8 is designed and implemented.

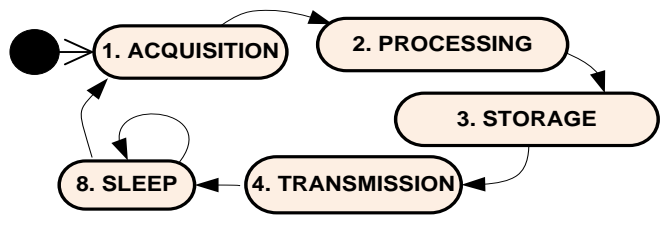

Figure 7. Finite state machine of the sensor node firmware

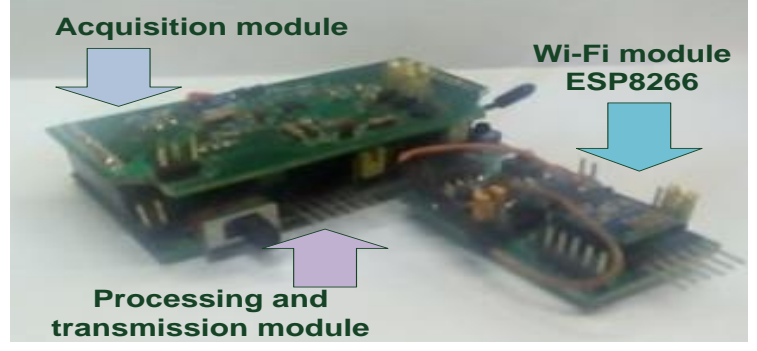

Figure 8. Design (left) and implementation (right) of the sensor node

\subsection{System Implementation Scenario Outline}

To carry out the communication tests, three nodes each with a type of sensor from Table 1 were implemented. In the first scenario, ZigBee communication is tested together with a GPRS coordinator to send information to a server over the Internet. In the second scenario, Wi-Fi nodes are used which connect to an Access Point LinkSys WRT54G. Scheme of the test scenario shown in Figure 9.

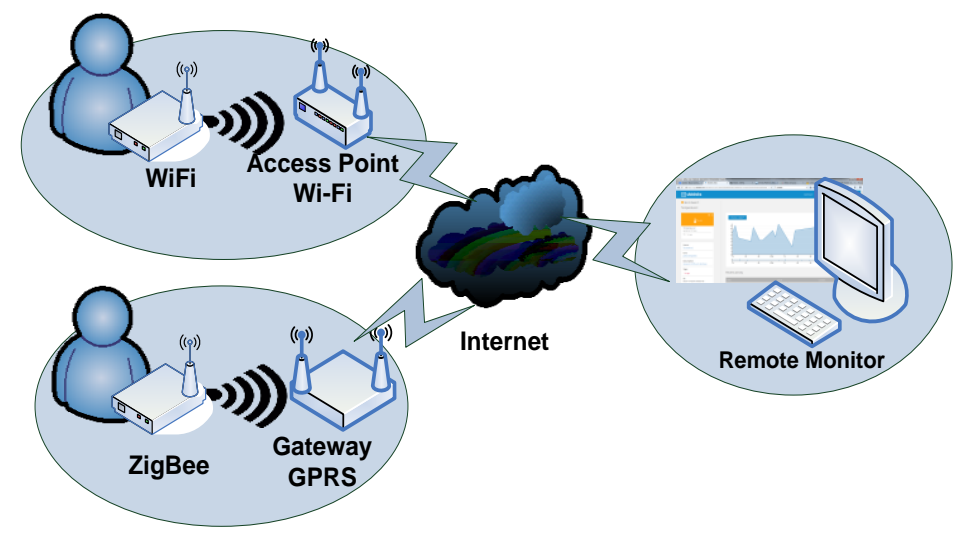

Figure 9. Scheme of the test scenario

In both scenarios, measurements are made of power consumption, transmission time and number of shipments made by modifying the period and amount of data sent, keeping the distance between the receiver and transmitter at 1 meter to avoid interference. 


\section{SYSTEM TESTS AND/OR RESULTS}

Using the 3 sensor nodes developed (each with one type of sensor) the current consumption measurements were made in each operating mode shown in Table 3. This table shows that the highest power consumption is provided by the Wi-Fi module, while the Xbee module has better performance in low power mode.

Table 3. Power Consumption of the Wireless Modules

\begin{tabular}{ccccc}
\hline & $\begin{array}{c}\text { Sleep } \\
(\mathrm{mA})\end{array}$ & $\begin{array}{c}\text { Run } \\
(\mathrm{mA})\end{array}$ & $\begin{array}{c}\text { Transmit } \\
(\mathrm{mA})\end{array}$ & $\begin{array}{c}\text { Peak Transmit } \\
(\mathrm{mA})\end{array}$ \\
\hline Xbee Series 2 & $<0.02$ & 18 & 40 & 45 \\
ESP8266 & $<0.1$ & 74 & 135 & 860 \\
\hline
\end{tabular}

The readings of the execution times of each process showed that the acquisition time varies proportionally to the sampling rate of the signal. In the case of transmission time, the ESP8266 module has a time that varies between 25 and $39 \mathrm{~ms}$ while the Xbee module changes according to the number of bytes to be transmitted, as shown in Table 4. Figure 10 shows that the average current consumption at each sensor node is calculated by multiplying the current consumption and run time at each process and dividing this result by the shipping period.

Table 4.Time of each process

\begin{tabular}{ccc}
\multicolumn{3}{c}{ Table 4.Time of each process } \\
\hline & Xbee Series 2 (ZigBee) & ESP8266 (Wi-Fi) \\
\hline Startup time & $0.1-0.2$ & 0.3 a $0.6 \mathrm{seg}$ \\
Transmission time & depends 1 byte*1 ms & $25-39 \mathrm{~ms}$ \\
Time of acquisition & varies according to the amount of data in both & cases \\
\hline
\end{tabular}

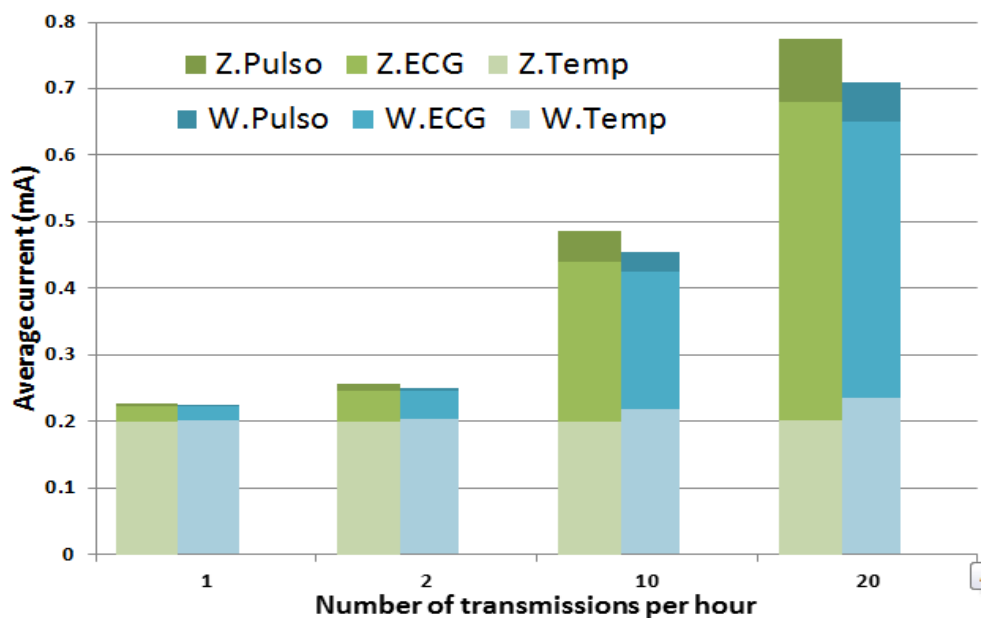

Figure 10. Average current consumption of the 3 sensor nodes

The number of bytes sent was 380 bytes in all cases (with the exception of the temperature sensor node) and it is observed that the Wi-Fi node consumes less current than the ZigBee node when the number of sends per hour decreases, but when it increases, the consumption of the ZigBee node exceeds the Wi-Fi node. In the case of the pulse sensor it consumes more current than the other nodes and this is due to the fact that the sensor's LED requires a longer stabilization time to acquire the samples.

Figure 11 shows the percentage of power consumed in the sleep mode, acquisition, processing and transmission stages of the ECG sensor node with transmission periods of 1, 10 and 20 times per hour and with 100, 300, 500 and 800 byte packets. The ECG sensor node was chosen to be represented in Figure 11 because it has a behavior comparable to the ZigBee node in current consumption. The upper part of Figure 11 shows that when 100 bytes of data are sent (in all cases) the percentage of current consumption in sleep mode 
is higher. When the data sent increases, so does the power consumption at the processing stage but to a lesser extent at the transmission stage.

The lower part of Figure 11 shows the power consumption distribution of the Wi-Fi node, noting that in Sleep mode the current percentage is very similar to the ZigBee node. The power consumption of the Wi-Fi node in Sleep mode increases for periods of 10 and 20 transmissions per hour being higher than that of the ZigBee node.

A comparison of the lifetime of the Wi-Fi and ZigBee ECG node can be seen in Figure 12 for a frequency of 20 shipments per hour. For 100 byte transmissions the Xbee node has a lifetime of 203 days and the Wi-Fi has a lifetime of 189 days. When the amount of data sent increases to 800 bytes, the lifespan of both types of nodes decreases but the Wi-Fi node performs better than the ZigBee when sending data over 300 bytes.
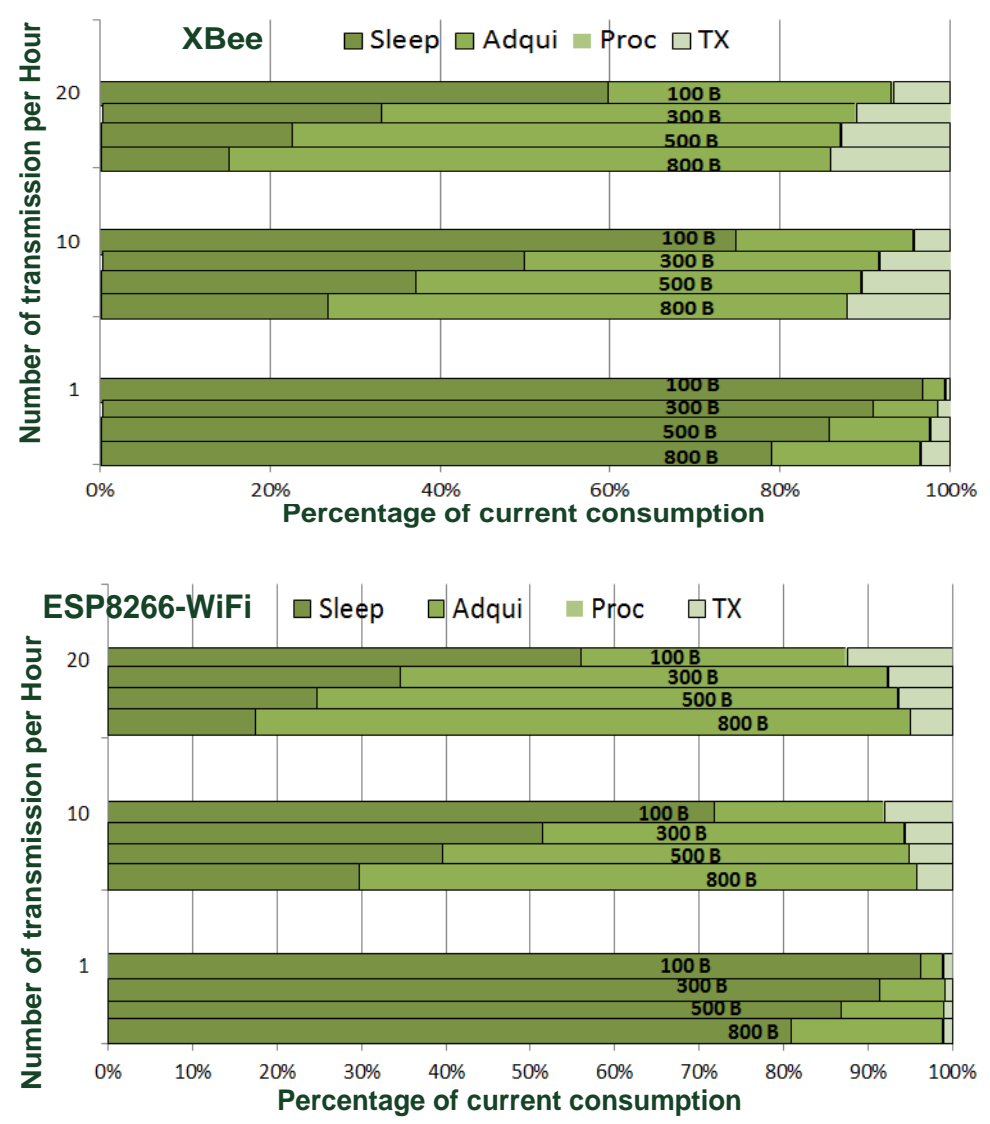

Figure 11. Percentage of current consumption in each process at sensor node Xbee (upper) and ESP8266 (lower)

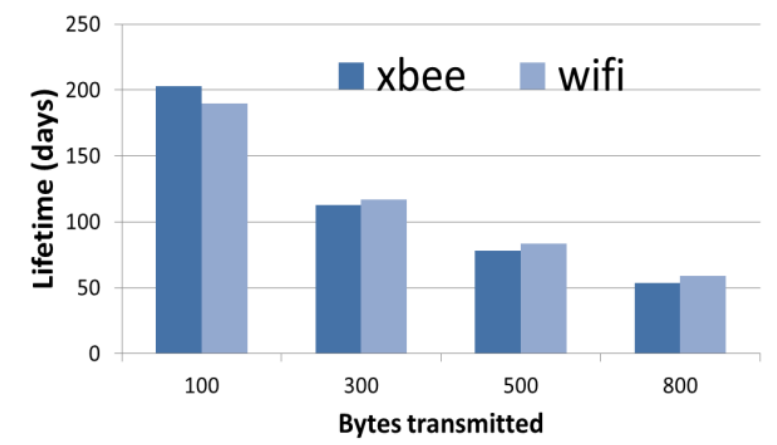

Figure 12. Lifetime of the ZigBee and Wi-Fi sensor node 


\section{CONCLUSION}

The sensors and wireless communication modules were chosen for their availability on the market, low power consumption and compatibility for use with a $3.3 \mathrm{~V}$ microcontroller. Communication test results show that the choice of current efficient wireless protocol and lifetime depends on the number of bytes sent and their transmission period which is related to the type of physiological sensor used.

The WiFi node had a longer lifetime than the ZigBee for periodic sendings with data transmissions of more than 300 bytes. Although it is observed that both nodes diminish in their life time, the Wi-Fi node manages to surpass slightly the ZigBee, therefore it is recommended to use it in implementations that require transmitting hundreds of bytes. In this way, it takes advantage of the features associated with connectivity, ease of installation in network infrastructures and the variety of mobile devices that use this protocol.

In the case of the ZigBee sensor node it has a longer lifetime when used for transmissions of less than 100 bytes (in the particular case of the ECG) and with periods of less than 2 sends per hour. One detail to consider is that the speed with which the data was sent to the ZigBee modules was through the serial UART protocol which limited the theoretical speed they could reach. This article is intended to develop future work related to evaluating other wireless communication modules and their transmission characteristics with other types of sensors such as images or audio.

\section{ACKNOWLEDGEMENTS}

This paper was developed in the Faculty of Electrical and Electronic Engineering, National University of Callao and the laboratories of INICTEL-UNI (National Institute of Research and Training in Telecommunications - National University of Engineering).

\section{REFERENCES}

[1] Betancur, Leonardo, "Redes de área corporal. Una perspectiva al futuro desde la investigación", Sistemas y Telemática, Vol.9 (16), pp. 11-30, March 2011.

[2] Kim Tae-Yoon, "Multi-hop wban construction for healthcare iot systems", IEEE International Conference on Platform Technology and Service, pp. 27-28, Abril 2015.

[3] ZigBee TM Networks, XBee TM Series 2 OEM RF Modules, Access: http://www.compel.ru/images/catalog/868/product-manual_XBee_Series2_OEM_RF-Modules_ZigBee.pdf

[4] SeeedStudio, ESP-12E Brief Spec, 2012. Access: http://www.seeedstudio.com/wiki/images/7/7d/ESP12E_brief_spec.pdf

[5] Ronald N. Meza, Ling A. Vilca, Percy Monroy Vilcahuaman, "ECG con diagnostico por sistema experto y comunicación GPRS”, Universidad Nacional de san Agustín, Perú, 2013.

[6] Brown, Brian H., et al. Medical physics and biomedical engineering. CRC Press, 1998.

[7] World Famous Electronics LLC, Pulse sensor datasheet, 2016 Access: https://pulsesensor.com

[8] Shivwanshi, Resham Raj, "Design and development of wireless sensor network for biomedical application", IEEE 2016 2nd International Conference on Advances in Computing, Communication, \& Automation (ICACCA), September 2016.

[9] Córdova Diana Milena Archila, Frey Alfonso Santamaría, "Estado del arte de las redes de sensores inalámbricos", Tecnología Investigación y Academia. 2013; 1(2).

[10] Londoño Jonathan Gallego Duque, Juan Diego Lemos Valdivieso, Alher Mauricio Hernández, "Diseño de un dispositivo portátil e inalámbrico para el monitoreo ambulatorio de la presión arterial no invasiva", Revista Politécnica. 2015; 6 (11): 121-131.

[11] Chauhan Jigar; Bojewar Sachin. "Sensor networks based healthcare monitoring system", En Inventive Computation Technologies (ICICT), International Conference on IEEE, 2016: 1-6.

[12] Joshi Jetendra, et al. "Health Monitoring Using Wearable Sensor and Cloud Computing”, Cybernetics Robotics and Control (CRC), International Conference on IEEE. 2016, 104-108.

[13] Puvaneshwari, S., Vijayashaarathi S. ,"Efficient monitoring system for cardiac patients using wireless sensor networks (WSN)", Wireless Communications, Signal Processing and Networking (WiSPNET), International Conference on IEEE, 2016. p. 1558-1561.

[14] Kolban Neil, “Kolban's Book on ESP8266”, Texas, USA, 2015, p. 1-317.

[15] Wang Vanessa, Salim, Frank Moskovits Peter, "The definitive guide to HTML5 WebSocket”, New York: Apress, 2013.

[16] Snajder Boris, et al, "Wireless sensor node modelling for energy efficiency analysis in data-intensive periodic monitoring”, Ad Hoc Networks, 2016. 49:29-41. 


\section{BIOGRAPHIES OF AUTHORS}

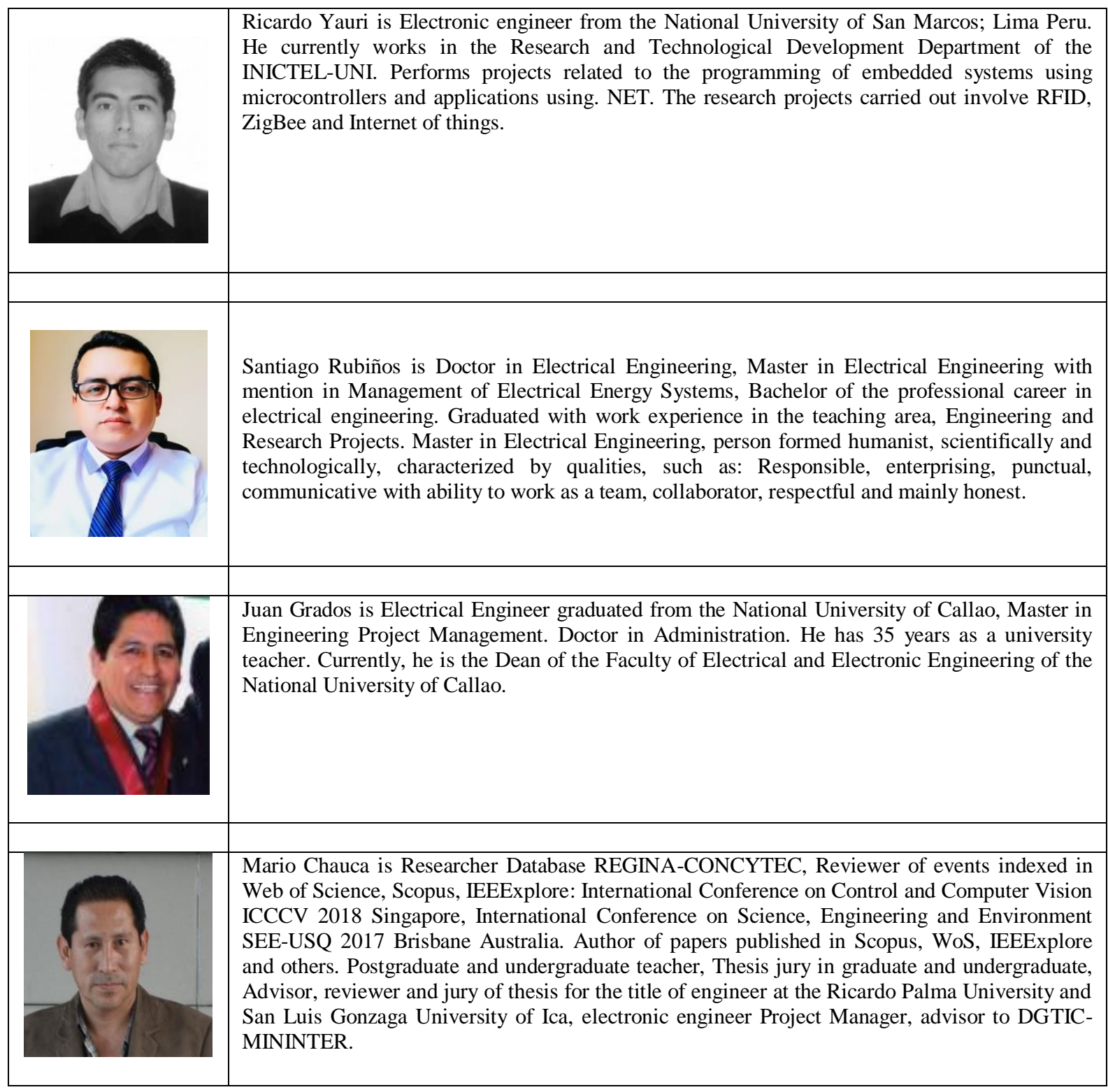

